\title{
Not all group members are created equal: heterogeneous abilities in inter-group contests
}

\author{
Francesco Fallucchi $^{1} \cdot$ Enrique Fatas $^{2,3,4}$ (D) $\cdot$ Felix Kölle $^{5} \cdot$ Ori Weisel $^{6}$
}

Received: 18 December 2018 / Revised: 5 August 2020 / Accepted: 3 September 2020 /

Published online: 10 December 2020

(c) The Author(s) 2020

\begin{abstract}
Competition between groups is ubiquitous in social and economic life, and typically occurs between groups that are not created equal. Here we experimentally investigate the implications of this general observation on the unfolding of symmetric and asymmetric competition between groups that are either homogeneous or heterogeneous in the ability of their members to contribute to the success of the group. Our main finding is that relative to the benchmark case in which two homogeneous compete against each other, heterogeneity within groups per se has no discernable effect on competition, while introducing heterogeneity between groups leads to a significant intensification of conflict as well as increased volatility, thereby reducing earnings of contest participants and increasing inequality. We further find that heterogeneous groups share the labor much more equally than predicted by theory, and that in asymmetric contests group members change the way in which they condition their efforts on those of their peers. Implications for contest designers are discussed.
\end{abstract}

Keywords Contests · Groups $\cdot$ Abilities $\cdot$ Heterogeneity $\cdot$ Experiments

JEL Classification $\mathrm{C} 72 \cdot \mathrm{D} 72 \cdot \mathrm{C} 92 \cdot \mathrm{H} 4$

\section{Introduction}

Many situations in social and economic life are characterized by rivalry and conflict between two or more competing parties. Warfare, socio-political conflicts, political elections, lobbying, $\mathrm{R} \& \mathrm{D}$ competitions, and promotion tournaments, are all examples of inter-group conflicts in which groups spend scarce and costly resources in order to compete with other groups. Within each competing group, group members

Electronic supplementary material The online version of this article (https://doi.org/10.1007/s1068 3-020-09677-5) contains supplementary material, which is available to authorized users.

Enrique Fatas

efatas@icesi.edu.co

Extended author information available on the last page of the article 
may differ with respect to a variety of characteristics such as preferences, resources, wealth, productivity, or motivation, which, in turn, can affect their ability and willingness to compete. Acknowledging that such within-group heterogeneity is the rule rather than the exception, a straightforward implication is that competing groups are rarely identical, and contests are typically not symmetric.

Examples abound. For instance, countries competing for access to natural resources or geopolitical influence will typically (if not always) differ regarding the degree of diversity in society, such as the distribution of income, education, or other sociodemographic characteristics. In the domain of organizations, firms often rely on interfirm alliances to compete with other firms or alliances (for example in the context of developing new products). Such alliances can be cross-function when partners contribute diverse and complementary resources, or same-function when firms have similar competencies (Amaldoss and Staelin 2010). The resulting competition can then be either symmetric (between two cross-function alliances or two same-function alliances) or asymmetric (between a cross-function and a same-function alliance). Similarly, within-firm contests between groups can occur in the context of performance-contingent payment schemes, such as paying bonuses to the best performing group(s) to increase productivity (Nalbantian and Schotter 1997; Bandiera et al. 2013). Within-group heterogeneity in such settings is only natural, as group members can have different skills or abilities. It follows that the competing groups themselves are also not necessarily similar, resulting in asymmetric competition. ${ }^{1}$

Despite these rather natural applications, the bulk of previous literature on contests has focused on situations in which symmetrical agents or groups compete against each other (see Dechenaux et al. 2015; Sheremeta 2017, for overviews). There is, however, a respectable (and growing) number of studies that have investigated the effects of various types of asymmetries, including group size (Abbink et al. 2010; Ahn et al. 2011), wealth (Rapoport et al. 1989; Hargreaves Heap et al. 2015), sharing rules (Kurschilgen et al. 2017), and the availability of communication (Cason et al. 2017) and punishment opportunities (Sääksvuori et al. 2011).

We contribute to this literature by experimentally testing how heterogeneity in players' ability to contribute to the group's success affects competition. In our systematic analysis, we study the effect of heterogeneity both within and between groups. For ease of exposition, we will use the terms homogeneous and heterogeneous to describe within-group structures (i.e., whether group members are similar or not), and symmetric and asymmetric to capture the relationship between the groups (i.e., whether competing groups are similar or not). In our setting, a high-ability person is more efficient in converting her effort to a contribution to the group than a low-ability person. This means that the marginal productivity, or the contribution/ effort ratio, of the high-ability person is higher: for each unit of invested effort, a high ability player contributes more to the group than a low ability player. Heterogeneity in this respect is only natural, as some group members may be stronger, smarter, or

\footnotetext{
1 The effect of heterogeneity on effort when individuals rather than groups compete for a reward has been analyzed by Chen et al. (2011) and Orrison et al. (2004), and the behavioural consequences of heterogeneity in team production by Hamilton et al. (2003) and Brandts et al. (2007, 2016).
} 
have better task-specific capabilities than others. As an illustration, think of a group of salespersons, with one member who is more talented, experienced, or is endowed with a more densely populated sales territory, than the others. The high ability individual has a higher marginal productivity in the sense that even if all group members exert the same effort (in terms of, e.g., hours worked or energy expenditure), she will contribute more to the group's success than her less able peers.

Previous studies modeled ability, or related concepts, in different ways. Sheremeta (2011b) manipulated players' valuation of the prize, i.e., some group members derive a higher payoff than others when the group wins the contest. ${ }^{2}$ Ryvkin (2011), in a theoretical model, and Brookins et al. (2015), in an experiment, consider within-group heterogeneity based on players' cost of effort; for some players, investing effort towards the group's success is costlier than for others. From an individual, self-interested, perspective, high abilities (as operationalized by us), high prize valuations, and low effort costs are all expected to increases players' inclination to contribute to their group. However, if players care not only about their own payoff, but also about the distribution of payoffs within the group (e.g., have a preference for equality) these different ways of introducing heterogeneity may differ in how they affect contributions. The reason is that when abilities are heterogeneous, equal efforts lead to equal payoffs; high-ability players' inclination to contribute is at odds with equality. When valuations or effort costs are heterogeneous, equal efforts result in un-equal payoffs; group members without higher prize valuations, or with lower effort costs, must contribute more than others for payoffs to be equal. Whether these differences in the modeling of ability matter behaviorally is ultimately an empirical question, which the current study can help answer.

We use a laboratory experiment to investigate the role of heterogeneity in abilities within and between groups. The major advantage of using a laboratory experiment is that it allows to tightly distinguish between a player's ability and her effort choice. In the field this is almost impossible to achieve, since typically only performancewhich is a function of both effort and ability (and noise) - can be observed. The laboratory setting further allows us to exogenously manipulate the composition of players within groups, which circumvents complicating factors such as self-selection that emerge in most field settings where groups form endogenously.

As a workhorse for studying group contests, we follow previous literature by using an experimental version of Tullock's contest game (Tullock 1980) in which two groups compete for a prize that is divided equally among all members of the winning party (Katz et al. 1990). We study this basic decision situation in three different treatments, in which we systematically vary the heterogeneity both within and between groups. In the first treatment, we study (commonly explored) symmetric contests between two homogeneous groups, in which all group members in both groups are equally able to compete. To study the pure effect of within-group heterogeneity, in the second treatment both competing groups are equally heterogeneous. Specifically, each group consists of one low-ability, one medium-ability, and

\footnotetext{
${ }^{2}$ Kölle (2015) shows, in a single group setting, that heterogeneity in valuations can lead to very different behavioral reactions than heterogeneity in abilities.
} 
one high-ability player. Importantly, we hold the average ability of group members constant compared to homogeneous groups, which consists of three medium-ability players. In the third and last treatment, we focus on the most interesting and natural situation in which the two competing groups differ from each other. To provide a clean comparison to the first two treatments, and to be able to investigate how conflict engagement depends on the opponent's group type (while holding constant the own group type), we examine an asymmetric contest between a homogeneous group and a heterogeneous group.

This set of treatments further distinguishes our study from the above-mentioned studies by Sheremeta (2011b) and Brookins et al. (2015). In both studies competing groups are always heterogeneous to a certain degree; in the latter, contests are always asymmetric. In contrast, our design includes the natural benchmark, featured in the bulk of the literature, of contests involving completely homogeneous groups as well as symmetric contests between heterogeneous groups. Including these situations allows to better identify and separate the role of within- and between-group heterogeneity. ${ }^{3}$

We find that while heterogeneity per se has no discernable impact on the degree of competition, asymmetry between groups leads to an intensification of conflict, with homogeneous and heterogeneous groups winning the contest equally often. One reason for this result is that players in heterogeneous groups contribute to the success of the group much more equally than predicted by a number of theories (see Sect. 2), which agree in stating that only high-ability players should contribute, while low- and medium-ability players should free-ride. ${ }^{4}$

Intra- and inter-group dynamics - the way individuals condition their behavior on the past behavior of others-help to further explain these results. We find that for homogeneous groups, asymmetric contests lead to increased conditional cooperation among group members, while no such effect is observed for heterogeneous groups. Furthermore, in heterogeneous groups players of different abilities condition their effort on that of their 'relevant' peer (see Croson et al. 2005, 2015; Kölle 2015). Low-ability players react to efforts by the medium-ability player but largely ignore the ones by the high-ability player. Medium-ability players, in contrast, react to both low- and high-ability players. The latter, in turn, react to the behavior of their medium-ability group member, but only in symmetric contests; when the contest is asymmetric they become "unconditional cooperators", as their contribution is not driven by the effort levels of their group members.

\footnotetext{
3 Relatedly, Bhattacharya (2016) studies the effects of differences in abilities and differences in costs across groups. Her study is different from ours, however, as she focuses on differences between groups in asymmetric contests (with one underdog and one favorite group), while we study heterogeneity both within and between groups without giving any party a comparative advantage.

4 These results are similar to those reported in Sheremeta (2011b) who finds that when players differ regarding their prize evaluation, in asymmetric contests more heterogeneous groups do not utilize their comparative advantage over less heterogeneous groups. Furthermore, similar to our results, he finds that group members share the burden of exerting effort much more equally than predicted by theory, although to a lesser extent than observed in our setup. A similar effect was observed by Brookins et al. (2015), who also find efforts to be much more similar than predicted by theory.
} 
Finally, we show that asymmetric contests are not only more intense, but also more volatile, suggesting that facing a group that is different from your own can lead to an increase in strategic uncertainty. As a result of the increased intensity and volatility, asymmetric contests have two detrimental effects for the participants as they decrease individual earnings and increase payoff inequality within groups. Taken together, our results show that heterogeneity in abilities significantly affects contest behavior only in the most natural setting where heterogeneity exists both within and between groups.

The rest of the paper is organized as follows. Section 2 presents the general setup of our group contests as well as theoretical predictions. In Sect. 3 we describe our experimental design and procedures in more detail. Section 4 summarizes our results. In Sect. 5 we discuss our findings and provide some behavioral rationale for the observed effects. Section 6 concludes.

\section{Model and predictions}

Consider the following inter-group contests for public goods by Katz et al. (1990). Let there be $n=2$ groups of $m \geq 1$ risk-neutral players each, competing to win a prize $m V$. All group members are endowed with the same amount of resources (e.g., time) $w$, which they can either use to help the own group win the contest, or they can use it for themselves (e.g., for an alternative private activity). Let $e_{i, j} \geq 0$ represent the effort (resources) spent by player $i$ in group $j$. Importantly, players may differ in their ability to contribute to their group activity; a high-ability player is better able than a low-ability player in converting her effort to an actual contribution to the group. That is, her contribution to the group output is given by $x_{i, j}=e_{i, j} \cdot \alpha_{i, j}$, where $\alpha_{i, j}$ is the ability of player $i$ in group $j$ determining her marginal productivity of effort. We assume that individual group members' efforts are perfect substitutes, i.e., total effort of group $j$ is given by $E_{j}=\sum_{i=1}^{m} e_{i, j}$ and the total contribution of group $j$ is given by $X_{j}=\sum_{i=1}^{m} \alpha_{i, j} e_{i, j}$. Following Tullock (1980), the probability of group $j$ winning the contest and securing the prize is given by the following contest success function:

$$
p_{j}\left(X_{1}, X_{2}\right)= \begin{cases}\frac{X_{j}}{X_{1}+X_{2}} & \text { if } X_{1}+X_{2}>0 \\ \frac{1}{2} & \text { otherwise. }\end{cases}
$$

When a group wins, each member of the winning group receives an equal share of the prize, $V .^{5}$ The expected payoff of player $i$ in group $j$ is then given by

\footnotetext{
${ }^{5}$ Equal sharing rules are a common way to distribute bonuses within teams, e.g., in sports competitions, especially when efforts are not fully observable or not verifiable (see Kurschilgen et al. 2017, for a study on the effect of sharing rules in inter-group competition).
} 


$$
\pi_{i, j}\left(x_{i, j}, X_{1}, X_{2}\right)=w-e_{i, j}+\frac{X_{j}}{X_{1}+X_{2}} V
$$

In our experiment (see Sect. 3), we consider two different type of groups, which can be either homogeneous or heterogeneous in the composition of the ability of their members. It follows that three types of inter-group contests can arise: two symmetric contests where both groups share the same internal composition of their members' ability (either homogeneous or heterogeneous), and one asymmetric contest with a homogeneous group competing with a heterogeneous one. The different contests may lead to different predicted outcomes. We describe below the resulting equilibria predicted under three distinct assumptions: players maximize either their own earnings; players maximize the joint-profit of their own group; or players maximize the difference in earnings between their own and the competing group.

Under the standard assumption of purely self-interested individuals, following Konrad (2009), if all players in all groups are equally able to contribute to the group output, i.e., $\alpha_{i, j}$ is the same for all players (symmetric homogeneous contest), there is a unique equilibrium prediction for the total group effort that is the same as in a two-player contest and equal to $E_{1}=E_{2}=\frac{V}{4}$. Theory, however, remains silent about the behavior of individual group members: any combination of efforts that add up to $\frac{V}{4}$ constitutes an equilibrium. When both groups are heterogeneous but identical (symmetric heterogeneous contest), the prediction about the group effort level does not change, but there is a clear-cut prediction for the individual efforts. As contributions to the group are perfect substitutes and costs of effort are linear, in equilibrium only the member with the highest ability in each group should exert effort, while the other group members should free ride (see Baik 2008, for a similar result when group members differ with regard to the evaluation of the prize).

When groups differ with respect to the most able group member (asymmetric contests), the group contest reduces to an asymmetric contest between the most able group members in each group. In the following, we denote the ability of the most able member within each group by $\bar{\alpha}_{j}$. The solution is then given by simultaneously solving the two reaction functions of the optimal efforts of the two players, yielding $E_{1}=\frac{\left(\bar{\alpha}_{1} \bar{\alpha}_{2} E_{2} V\right)^{\frac{1}{2}}-\bar{\alpha}_{2} E_{2}}{\bar{\alpha}_{1}}$ and $E_{2}=\frac{\left(\bar{\alpha}_{1} \bar{\alpha}_{2} E_{1} V\right)^{\frac{1}{2}}-\bar{\alpha}_{1} E_{1}}{\bar{\alpha}_{2}}$. By denoting $\alpha=\bar{\alpha}_{1} / \bar{\alpha}_{2}$ as the relative ability between the most able player of both groups, the resulting unique symmetric Nash Equilibrium is given by $E_{1}=E_{2}=\frac{\alpha V}{(1+\alpha)^{2}}$, which for $\alpha \neq 1$ is strictly smaller than $\frac{V}{4}$. As a result, effort is predicted to be lower in asymmetric than in symmetric contests (see also Fonseca 2009, for a similar result investigating heterogeneity between players in individual contests).

Experimental evidence from group contests typically shows a departure from standard predictions, with a general tendency of over-dissipation (see Sheremeta 2017, for an overview). Several explanations have been put forward to explain such over-investments by groups. Some studies have argued that agents derive some nonmonetary joy of winning (Sheremeta 2010), while others explain this finding by assuming that players make mistakes and are only boundedly rational (Lim et al. 2014). Further studies argue that agents are motivated not only by self-interest, but 
also care about the payoffs of others. In particular, agents may be motivated by joint profit maximization, i.e., they strive to maximize the sum of payoffs within the own group (Leibbrandt and Sääksvuori 2012), or by relative payoff maximization/parochial altruism (i.e., the display of altruism towards in-group members along with hostility towards out-group members (Bernhard et al. 2006; Choi and Bowles 2007; Abbink et al. 2010)), leading them to maximize the difference between the own and the other group's payoff. Applying the last two concepts to our setting, the following qualitative predictions across treatments can be derived.

When players try to maximize joint payoffs, the objective function is given by $m w+\frac{X_{j}}{X_{1}+X_{2}} m V-E_{j}$. In this case, in symmetric contests the predicted effort is equal to $E_{1}=E_{2}=\frac{m V}{4}$, while in asymmetric contests aggregate effort is predicted to be $E_{1}=E_{2}=\frac{\alpha m V}{(1+\alpha)^{2}}$. Hence, while compared to the standard prediction of purely selfish players effort levels are predicted to be higher, it still holds that effort should be lower in asymmetric than in symmetric contests, and that in heterogeneous groups only high ability players should exert any effort.

If, instead, group members are motivated by parochial altruism, they strive to maximize the difference between their own and the other groups' payoff, $\left(\frac{X_{1}}{X_{1}+X_{2}} m V-E_{1}\right)-\left(\frac{X_{2}}{X_{1}+X_{2}} m V-E_{2}\right)$. In this case, the total effort exerted by each group in symmetric contests will be $E_{1}=E_{2}=\frac{m V}{2}$, which is higher than predicted efforts in asymmetric contests, given by $E_{1}=E_{2}=\frac{2 \alpha m V}{(1+\alpha)^{2}}$. Both of these effort levels are higher than the ones predicted under the assumption of pure self-interest and joint payoff maximization. Yet, as before it holds that in heterogeneous groups only high ability players should be active, while in homogeneous groups any combination of efforts leading to the predicted aggregated effort is an equilibrium.

To summarize, while the three different assumptions about agents' objective function lead to level differences in the predicted effort levels, they all share some common qualitative characteristics. First, irrespective of the type of contest (symmetric or asymmetric) homogeneous and heterogeneous groups are predicted to exert the same level of aggregate effort. Second, within heterogeneous groups only the member with the highest ability should exert any positive effort (the others should free ride), while in homogeneous groups there is a continuum of optimal effort combinations. Third, total effort is predicted to be higher in symmetric compared to asymmetric contests.

In our experiment (see below) we empirically test each of these predictions. With regard to the predicted effort levels within heterogeneous groups, we expect behavior to deviate from theory as it predicts an extreme distribution of labor, which, in turn, creates substantial inequality within groups. There is now ample evidence from a variety of contexts that many people care about the relative distribution of outcomes (see e.g., Fehr and Schmidt 1999; Bolton and Ockenfels 2000; Sobel 2005; Fehr and Schmidt 2006). Applied to our context, if players dislike inequality within groups they have an incentive to match their group members' efforts, as payoff equality can only be obtained if all group members exert the same level of effort, irrespective of their ability. For heterogeneous groups, this is in stark contrast to the theoretical predictions above which 
state that only the high ability player should contribute. Hence, if players are also motivated by fairness considerations within groups, we should expect a more equal distribution of labor in heterogeneous groups. In Sect. 5 we address this point more formally and discuss how inequity concerns can rationalize our empirical findings. ${ }^{6}$

\section{The experiment}

Our experimental game is based on the model described above. Participants were randomly divided into three-person groups $(m=3)$. Each group was then matched with another, randomly selected, group $(n=2)$, to compete for a prize in 45 consecutive periods using a partner-matching protocol. We chose this fixed matching protocol as many field settings are characterized by repeated interactions among the same agents. Furthermore, we chose a rather long repetition of 45 rounds because previous research has documented pronounced learning effects in group contests (Fallucchi et al. 2013), and we were not only interested in contest behavior in the short-run, as most previous literature, but also in how heterogeneity and asymmetry affect competition in the long-run, once participants had sufficient time to learn.

The prize was 300 points, to be shared equally among the members of the winning group, irrespective of their individual efforts (i.e., for each member $V=100$ ). In each period, each group member received an endowment of 100 tokens $(w=100)$, which they could either use for their own private consumption or use to exert effort $e_{i} \in\{0,1, \ldots, 100\}$ to increase the probability of the group winning the contest. There were three types of players, low-ability, medium-ability, and high-ability, which differed in the effectiveness of their effort. Homogeneous groups consisted of three medium-ability players, and; heterogeneous groups consisted of one low-ability player, one mediumability player, and one high-ability player. ${ }^{7}$ Each token spent by a low-, medium-, or highability player yielded a contribution of one, two, or three to the group, respectively (i.e., $\alpha_{\text {low }, j}=1, \alpha_{\text {medium }, j}=2, \alpha_{\text {high, }}=3$ ). Player types were assigned randomly and remained constant throughout the experiment. Importantly, both homogeneous and heterogeneous groups have the same total endowment (300 tokens) and the same strategy space (contributions between 0 and 600). Cross matching these two group types yields two symmetric contests and one asymmetric contest, for a total of three experimental treatments: Symmetric-homogeneous, Symmetric-heterogeneous, and Asymmetric.

The Experiment was conducted at LabSi (University of Siena) using Z-Tree (Fischbacher 2007). A total of 258 participants were recruited for 15 sessions (13 with 18 participants and 2 with 12 participants each) resulting in 15 independent observations in symmetric homogeneous contests, 14 in symmetric heterogeneous contests, and 14 in asymmetric contests. At the beginning of each session, written instructions were handed out to participants and read aloud by the experimenter. After that, and before

\footnotetext{
6 See Kölle et al. (2016) for a formal analysis of these effects in a related setting.

7 In the instructions given to participants we avoided loaded terms like ability. Instead, low-, medium-, and high-ability players were given the labels Blue, Red, and Green, respectively. For an English translation of the instructions, see Appendix B in Electronic Supplementary Material.
} 
the start of the experiment, participants had to correctly answer a set of control questions to ensure correct understanding of the incentives and structure of the game. At the end of each period, players received detailed information about the effort, contributions, and earnings of their group members (sorted in descending order of efforts). They were further informed about the total contribution of their own group and the opponent group, as well as the outcome of the contest (see Appendix B in Electronic Supplementary Material for a screenshot of the feedback screen). At the end of the experiment, participants were paid their earnings in cash. Sessions lasted between 50 and $60 \mathrm{~min}$, and participants earned on average around $€ 8.50$.

Table 1 summarizes our experimental design as well as the theoretical predictions for each of the three conditions. Note that the predictions for parochial altruism (players try to maximize the payoff difference between both groups) slightly differ from those derived in Sect. 2, because effort levels were capped at each individual's endowment $(w=100)$, while in Sect. 2 there was no such constraint. While this cap does not change the prediction that in heterogeneous groups only the high-ability player should exert any effort, predicted group efforts in asymmetric contests are no longer the same (for both homogeneous and heterogeneous groups).

\section{Results}

We divide the presentation of our results into four subsections. In Sect. 4.1 we provide an overview of the main treatment differences. In Sects. 4.2 and 4.3 we focus in more detail on individual, ability- and group-specific behavior. In Sect. 4.4 we discuss the implications of the observed behavior on efficiency and inequality.

\subsection{The effects of contest type on the degree of competition}

Figure 1 summarizes the contest behavior in all three treatments, Symmetric homogeneous, Symmetric heterogeneous, and Asymmetric. The left panel shows the total average group efforts over all periods. The results reveal a clear pattern. We observe the lowest effort levels in symmetric-homogeneous contests in which two homogeneous groups compete against each other. Aggregated over all periods, group efforts amount to 67.2 (out of 300) tokens on average. When both groups are heterogeneous (symmetric-heterogeneous contest), group efforts moderately increase by about $19 \%$ to 79.8 tokens, but this effect is not statistically significant (Mann-Whitney U tests, $p=0.252$ ). In asymmetric contests (with one homogeneous and one heterogeneous group), in contrast, competition significantly intensifies. On average, group efforts amount to 93.3 tokens, which is $39 \%$ higher compared to our benchmark case with two homogeneous groups (Mann-Whitney U test, $p=0.057) .^{8}$

\footnotetext{
${ }^{8}$ In line with results from previous studies on group lottery contests (see Sheremeta 2017, for an overview), we find that effort levels in all treatments are way above the standard selfish equilibrium prediction $(+169 \%$ in symmetric-homogeneous contests, $+219 \%$ in symmetric-heterogeneous contests, and $+288 \%$ in asymmetric contests; Wilcoxon Signrank tests, all $p<0.001$ ). Relative to the predictions
} 


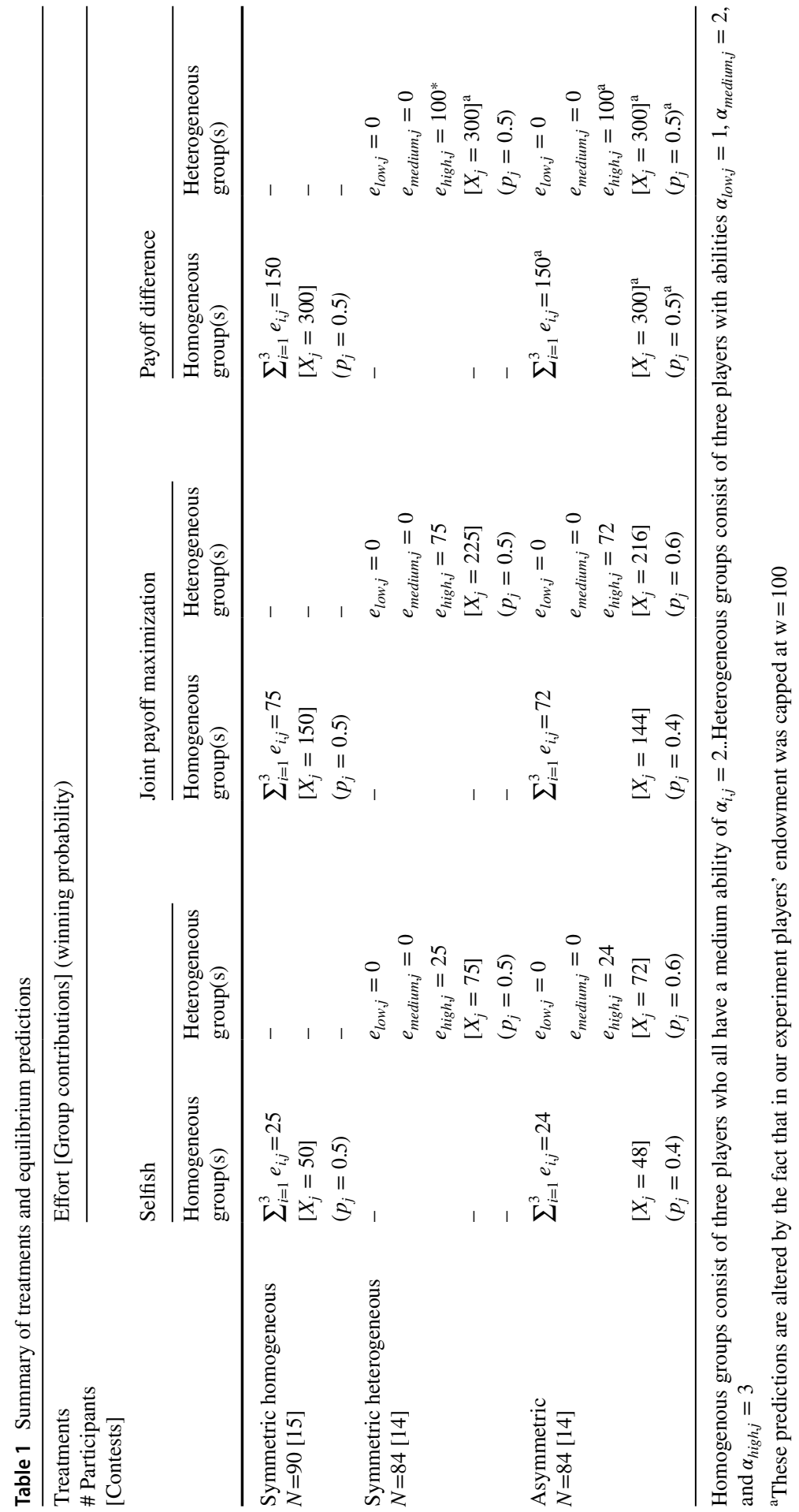


To further test the significance of these results, we run multilevel linear mixedeffects regressions that take into account the inter-dependency of observations (repeated observations of individuals that are nested within a contest of two competing groups). The results are shown in Table 2. In line with the results from the nonparametric tests, model (1) reveals that while the differences in efforts between Symmetric homogeneous and Symmetric heterogeneous are not significant $(p=0.283)$, efforts in Asymmetric are is significantly higher than in symmetric homogeneous contests $(p=0.028)$. We summarize these findings in our first result:

Result 1 It is mainly heterogeneity between groups-but not within groups-which leads to a significant intensification of conflict.

The right panel of Fig. 1 depicts the dynamics of group efforts over time (divided into 5-period blocks). As is apparent from this figure, in line with previous results (e.g., Abbink et al. 2010; Sheremeta 2010, 2011a; Fallucchi et al. 2013; Brookins and Ryvkin 2014), in all treatments we observe a significant downward trend of efforts. This decay is somewhat more pronounced in the asymmetric contest. To test for the significance of this observation, in model (2) of Table 2 we include a Period variable as well as interaction terms with this variable and the two treatment dummies (with symmetric homogeneous contests as the baseline category). The results corroborate the visual impression of a steeper negative time trend in the asymmetric contest, as indicated by the significant negative coefficient of the Period $\times$ Asymmetric interaction term $(p<0.001)$. At the same time, the results reveal that the dynamics in the two symmetric contests are very similar to each other, as the coefficient of the Period $\times$ Symmetric-heterogeneous interaction term is close to zero and not significant $(p=0.295)$. Taken together, these results suggest that the differences in group efforts between the asymmetric and the two symmetric treatments slightly reduce over time.

To directly test whether our treatment differences are driven by differences in the initial phase of the game or whether they also remain after participants had sufficient learning opportunities, we divide our data into two parts, the short-run (periods $1-20$ ) and the long-run (periods $21-45) .{ }^{9}$ We find that the differences in group

\footnotetext{
Footnote 8 (continued)

based on parochial altruism (maximizing payoff difference between groups), on the contrary, we observe effort levels that are lower than this benchmark; group efforts in Symmetric homogeneous, Symmetric heterogeneous, and Asymmetric amount to only 45\%, 80\%, and 75\%, respectively, of the predicted level (Wilcoxon Signrank tests, $p<0.001, p=0.042$, and $p=0.004$, respectively). It seems that our aggregate results are best captured by the predictions of joint payoff maximization; the deviations from the predicted levels are small and not significant (Symmetric homogeneous: $-10 \%, p=0.303$; Symmetric heterogeneous: $+6 \%, p=0.761$; Asymmetric: $+29 \%, p=0.091)$. Note, however, that none of these models can capture the comparative statics we observe across treatments, i.e., they cannot explain why effort increases when the contest involves heterogeneous groups. Furthermore, as we will show below, none of these models can capture the way efforts are distributed within each group.

9 We chose this cut-off value as 20 periods is a typical length in previous literature (see e.g. Abbink et al. 2010). Our results are robust, however, when using different cut-off values for defining the short-run, e.g., 15 or 25 periods.
} 


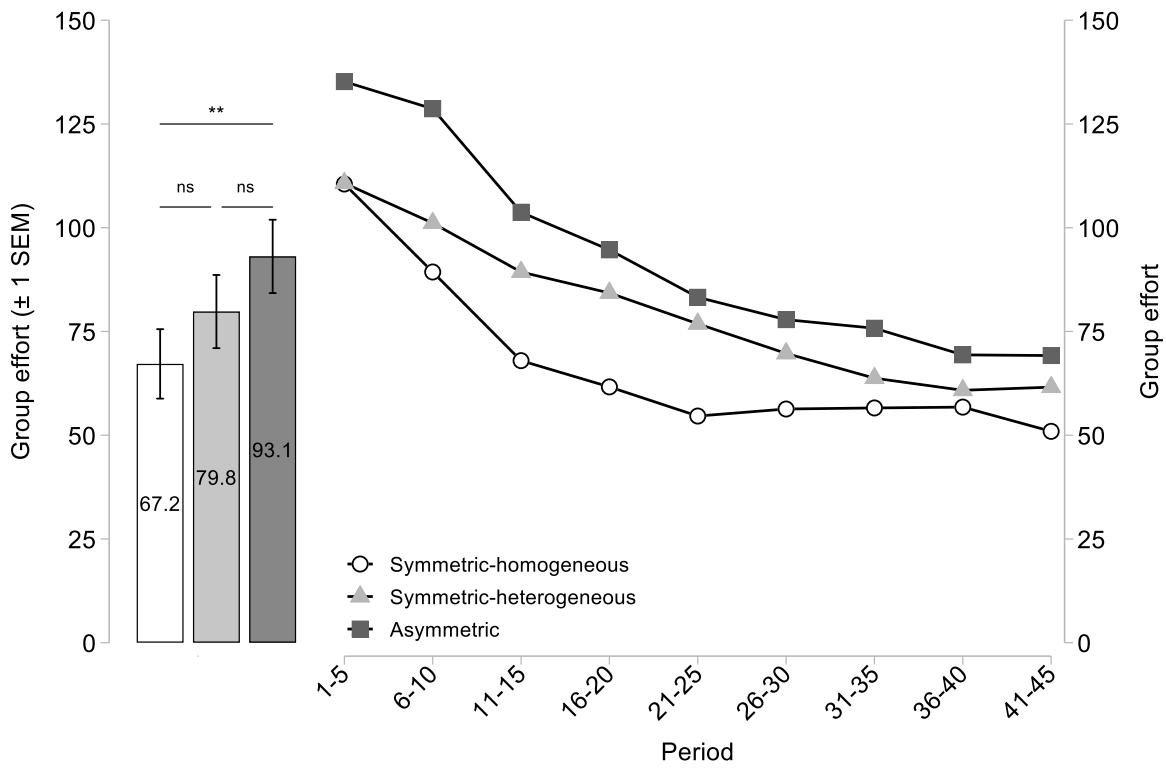

Fig. 1 Left panel: Group effort averaged over all periods ( \pm one standard error of the mean (SEM)). Right panel: Group effort by period (in blocks of 5). $*^{*} p<0.05$ according to multilevel linear mixedeffects regressions (as reported in model (1) in Table 2)

effort between the Asymmetric and the Symmetric homogenous treatment are statistically significant, both in the short-run and in the long-run (Mann-Whitney U tests: $p=0.029$ and $p=0.063$, respectively). The differences in efforts between these two types of contest, albeit becoming somewhat smaller over time, are thus a robust phenomenon that is not an artefact of initial noise or inexperience of participants. None of the other comparisons of group efforts between treatments yield significant results, neither in the short nor in the long-run (all $p>0.227$; see Table A1 in Electronic Supplementary Material in Appendix A for an overview).

In addition to the effect on the overall level of conflict, the type of contestsymmetric or asymmetric - also has an effect on the volatility of competition, both between and within groups. To measure between-group volatility, we calculate the absolute difference in group efforts between the two competing groups in a given period. We find that in asymmetric contests the average absolute difference in group efforts is substantially and significantly higher than in symmetric contests (47.2 vs. 37.4 (+26\%); Mann-Whitney U-test, $p=0.047)$, while no such difference is observed between the two symmetric contest treatments (Symmetric-homogeneous: 38.0, Symmetric-heterogeneous: 36.9; Mann-Whitney U-test, $p=0.561) .^{10}$

\footnotetext{
10 This effect is particularly pronounced in later periods. In particular, while in symmetric contests volatility significantly decreases over time (from 41.2 in periods $1-20$ to 34.4 in periods $21-45$; Wilcoxon Signrank test, $p=0.033$ ), in asymmetric contests it remains at a similarly high level throughout all periods (47.0 in periods $1-20$ and 47.4 in periods $21-45$; Wilcoxon Signrank test, $p=0.952$ ).
} 
Table 2 Group effort by treatment

\begin{tabular}{lll}
\hline Dependent variable: group effort & $(1)$ & $(2)$ \\
\hline Symmetric-heterogeneous & 12.612 & 14.079 \\
1 if Treatment=Symmetric-heterogeneous, O otherwise & $(11.758)$ & $(11.842)$ \\
Asymmetric & $25.895^{* *}$ & $37.180^{* * *}$ \\
1 if Treatment=Asymmetric, O otherwise & $(11.758)$ & $(11.842)$ \\
Period & & $-1.221^{* * *}$ \\
& & $(0.042)$ \\
Period x Symmetric-heterogeneous & & -0.064 \\
& & $(0.061)$ \\
Period x Asymmetric & & $-0.491^{* * *}$ \\
& & $(0.061)$ \\
Constant & $67.191^{* * *}$ & $95.278^{* * *}$ \\
& $(8.170)$ & $(8.228)$ \\
Test: Symmetric-heterogeneous=Asymmetric & $p=0.267$ & $p=0.059$ \\
Random intercepts: & & \\
Contest & Yes & Yes \\
Group & Yes & Yes \\
Observations & 3870 & 3870 \\
\hline
\end{tabular}

Multilevel linear mixed-effects models using random intercepts for matching groups (a contest between two groups) and groups. Numbers in parentheses indicate standard errors. Significance levels $* p<0.1$, $* * p<0.05, * * * p<0.01$

To measure within-group volatility, we calculate how much groups change their efforts (in absolute terms) from one period to the other. When both groups are of the same type, the average change in group effort between two consecutive periods is 26.3 and 24.8 , for symmetric-homogeneous and symmetric-heterogeneous contests, respectively (Mann-Whitney U-test, $p=0.533$ ). In asymmetric contests, this measure amounts to 32.4 tokens, significantly higher than in symmetric contests $(+27 \%$, Mann-Whitney U-test, $p=0.016) .{ }^{11}$

Overall, these results suggest a substantially increased degree of volatility when contests are asymmetric, even after participants have gained plenty of experience in the game. A possible explanation for this result is that when facing a group different from the own, it is harder to put oneself into the shoes of others and, hence, predict the opponent's behavior. As a consequence, strategic uncertainty increases, making behavior less stable. We summarize these findings in our second result:

\footnotetext{
${ }^{11}$ Similar to between-group volatility, we find that in symmetric contests within-group volatility significantly decreases from 28.2 in periods $1-20$ to 23.5 in periods 21-45 (Wilcoxon Signrank test, $p=0.002$ ), while in asymmetric contests no such time trend is observed (33.8 in periods $1-20,31.4$ in periods 21-45; Wilcoxon Signrank test, $p=0.326$ ).
} 
Result 2 In asymmetric contests there is a substantial increase in the volatility of competition, both within and between groups.

\subsection{Group- and type-specific behavior}

To understand what drives the increase in conflict expenditures in the asymmetric contest, we now zoom into group-, type-, and individual-specific behavior. First, we observe that both homogeneous and heterogeneous groups compete more aggressively in asymmetric contests, i.e., when they face a group different from their own. As shown in Fig. A1 in Electronic Supplementary Material in Appendix A, this effect is particularly pronounced for homogeneous groups, who, relative to the symmetric case, significantly increase their efforts by $45 \%$ from 67.2 to 97.5 tokens (Mann-Whitney U test, $p=0.026$; linear mixed effects model, $p=0.023$; see model (1) in Table A2 in Electronic Supplementary Material in Appendix A). In heterogeneous groups, effort also increases in asymmetric contests, but the effect is much smaller ( $+11 \%$, from 79.8 to 88.7 tokens) and not statistically significant (Mann-Whitney U test, $p=0.482$; linear mixed effects model, $p=0.452$; see model (2) in Table A2). ${ }^{12}$ Hence, it seems that mainly homogeneous groups suffer from the asymmetric contest, a point we will come back to in Sect. 4.4.

As a consequence, when comparing effort levels between the two different group types, we find that while in symmetric contests we observed higher effort levels in heterogeneous than in homogeneous groups, in asymmetric contests the opposite pattern is observed. Yet, in both cases the differences between the two group types are relatively small and not significant (Symmetric contests: -12.6, Mann-Whitney $\mathrm{U}$ tests, $p=0.252$; Asymmetric contests: +8.8 , Wilcoxon Signrank test, $p=0.583$ ), indicating (again) that it is the asymmetry between groups rather than the heterogeneity within groups that leads to an intensification of conflict. Interestingly, contrary to the theoretical predictions, we find that in asymmetric contests homogeneous groups even outperform heterogeneous groups in terms of actual contributions, i.e., effort $\times$ ability (195.0 vs. 183.3). As a result, homogeneous groups win the contest in $49.8 \%$ of the cases, significantly more often than the $40 \%$ predicted by standard theory (Wilcoxon Signrank test, $p=0.005$ ). ${ }^{13}$

To shed more light on these findings, in the following we take a closer look at behavior at the individual level. Recall that heterogeneous groups consist of players of different abilities - one low-, one medium-, and one high-ability player-and that all theories considered in Sect. 2 predict that only the high-ability player exerts effort, while the other players are predicted to completely free ride. Our empirical

\footnotetext{
12 This is reminiscent of the results by Hargreaves Heap et al. (2015) who find that when competing groups differ with regard to their endowment, in asymmetric contests between a poor and a rich group, the former responds doggedly by contributing more than in symmetric contests between two poor groups, at least when inequality is not too extreme.

13 A similar finding has been reported by Sheremeta (2011b), who finds that in asymmetric contests between groups that differ with regard to the group members' valuation of the prize, contrary to standard predictions the group with the member of the highest prize valuation does not win the contest more often than their counterpart.
} 
results are in stark contrast with these predictions. In symmetric contests, average efforts of low-, medium-, and high-ability players are practically identical, accounting for 33\%, 34\%, and 33\%, respectively, of the group's overall effort. Moreover, when ranking players based on their total efforts over all rounds, we find that only in one third of all groups the top contributor is also the player with the highest ability (compare Table A3 in Electronic Supplementary Material in Appendix A).

In asymmetric contests, this picture slightly changes. The efforts of low-, medium-, and high-ability players now account for 30\%, 33\%, and 37\%, respectively, of the group's overall effort. This indicates that the overall increase in effort in heterogeneous groups is driven to a large extent by the high ability types who increase their effort by 6.1 tokens, compared to only 1.8 and 0.9 tokens by low- and mediumability types (see also Fig. A2 in Electronic Supplementary Material in Appendix A). Still, in only $43 \%$ (6 out of 14) of all groups the high-ability group member is the top contributor. In the remaining groups, in most cases it is the medium-ability player (7 out of 14 ) rather than the low-ability player (1 out of 14) that take over the leading role as the top contributor (compare Table A3 in Electronic Supplementary Material in Appendix A). While on the one hand these results indicate that in asymmetric contests high-ability players take over some more responsibility by exerting more effort, on the other hand even in this case the distribution of efforts between the different ability types is far from the theoretical predictions stating that only high ability players should exert any effort (see Sheremeta 2011b, and Brookins et al. 2015 , for similar findings in related contexts). Instead, the data suggests that there is a strong norm of equal efforts, irrespective of the players' abilities, which might be due to fairness or inequity concerns within the group, as equality in earnings can only be achieved when all group members exert the same level of effort. We will come back to this point in Sect. 5, where we discuss how inequity concerns affect the incentives to exert effort within groups in much more detail.

To put the distribution of efforts within heterogeneous groups into perspective, we compare it to the distribution of labor within homogeneous groups. Note that because homogeneous groups consist of three identical players (of medium ability), theory remains silent about how effort should be distributed between them; any combination of efforts that leads to the predicted level of group effort is an equilibrium (compare Sect. 2). It is hence interesting to see whether the two different group structures, homogeneous and heterogeneous, differ in how group members share the burden of exerting effort, or whether they follow similar behavioral rules when coordinating their actions.

Figure 2 displays the efforts (averaged over all rounds) of the top, medium, and low contributors, separately for each group and contest type. In the absence of any heterogeneity in behavior, there would be no difference between the efforts of the different contribution types. In contrast to that, our data reveals pronounced heterogeneity within groups. Averaged over all group and contest types, we find that top contributors account for $45 \%$ of the group's total effort, more than twice as much as low contributors (22\%). Interestingly, these shares are very similar for homogeneous and heterogeneous groups, despite the very different theoretical predictions. In symmetric contests, top, medium, and low contributors in homogeneous groups contribute $49 \%, 30 \%$, and $21 \%$, respectively, of the group's total effort, compared to $44 \%, 33 \%$, and $23 \%$ in heterogeneous groups. Similarly, in asymmetric contests the 


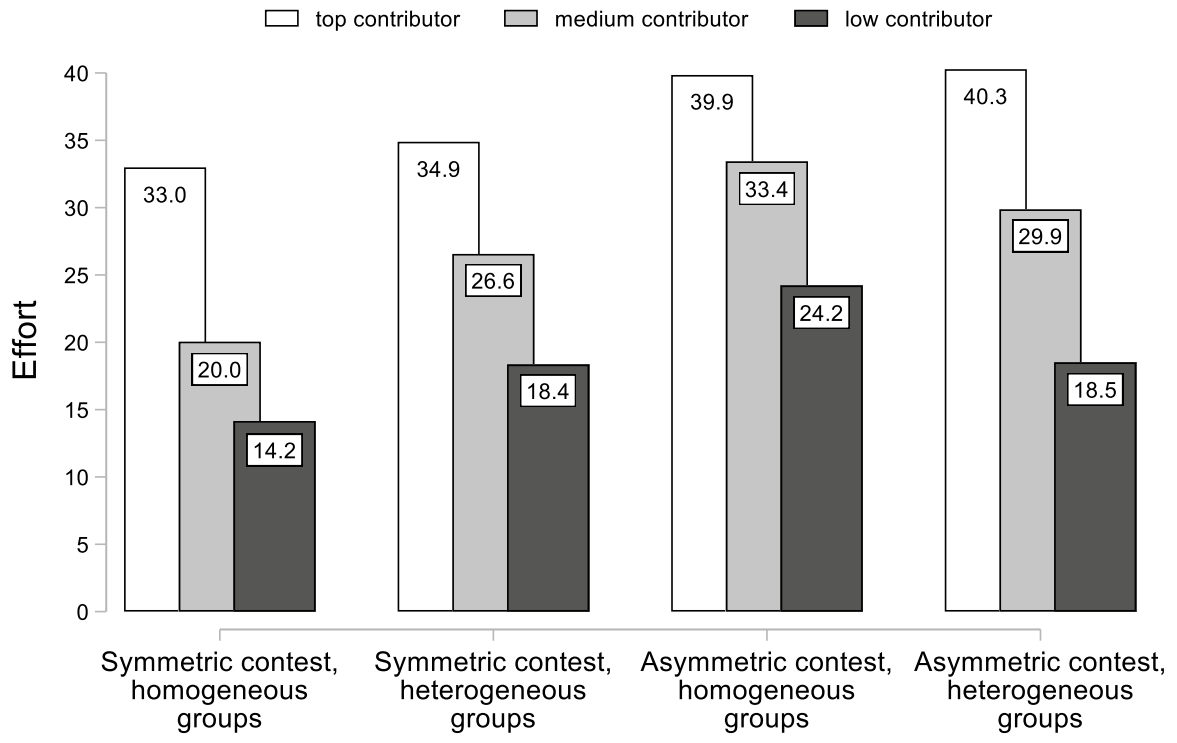

Fig. 2 Effort by group members ranked as top, medium, or low contributor, separately for each group and contest type

efforts of the top, medium, and low contributors in homogeneous groups account for $41 \%, 34 \%$, and $25 \%$, respectively, of the group's total effort, relative to $45 \%, 34 \%$, and $21 \%$ in heterogeneous groups. ${ }^{14}$

We summarize these findings in our third result:

\section{Result 3}

1. Homogeneous and heterogeneous groups both increase their efforts in asymmetric contests, but the effect is significant only for homogeneous groups.

2. The distribution of efforts in heterogeneous groups is very similar to the one in homogeneous groups, and much more equal than predicted by theory.

\footnotetext{
14 When comparing the behavior of the top, medium, and low contributors across symmetric and asymmetric contests, we find that efforts of all players increase when the contest becomes asymmetric. However, the magnitude of these effects differ somewhat between homogeneous and heterogeneous groups. In homogeneous groups we find that the strongest increase in efforts come from the medium contributors (+13.4 from 20.0 to 33.4; Mann-Whitney U test, $p=0.003$ ), followed by the low (+10.0 from 14.2 to $24.2, p=0.014$ ) and the top contributors (+6.9 from 33.0 to $39.9, p=0.121$ ). In heterogeneous groups, in contrast, the strongest increase in efforts comes from the top contributors (+6.4 from 49.9 to 40.3 ), followed by the medium ( +3.3 from 26.6 to 29.9 ) and the low contributors, who virtually do not change their exerted effort $(+0.1$ from 18.4 to 18.5$)$. Neither of these effects, however, is statistically significant (Mann-Whitney U test, all $p>0.217$ ), indicating that the increase in efforts is at an overall lower level compared to homogeneous groups (see also Fig. A1 in Electronic Supplementary Material in Appendix A).
} 
3. Heterogeneous groups do not utilize their predicted advantage over homogeneous groups; both types of groups win the contest equally often.

\subsection{Conditional cooperation and the inter-dependency of effort within and between groups}

To better understand these effects, in the following we provide a more detailed account of individual behavior by investigating the group dynamics of effort provision. In particular, we explore to what extent individual behavior is contingent on the lagged efforts of the other players - both in their own and in the opponent group - as well as on the type of contest. To this end, we run a set of multilevel mixed-effects regressions that explicitly take into account the inter-dependency of individual observations within a given (6-person) contest. The dependent variable is an individual's effort choice in period $t$. The main explanatory variables are the lagged average efforts of the two other members of the own group (Ingroup effort $t-1$ ), and the lagged average efforts of the opponent group (Outgroup effort $t-1$ ). To see whether behavior differs depending on the type of contest, we include interaction terms with an Asymmetric dummy which takes the value 1 if the contest is asymmetric, and 0 otherwise. For clarity and ease of interpretation, we use separate models for homogeneous (Model 1) and heterogeneous groups (Models 2). Because in heterogeneous groups different ability types might display different behavioral patterns, we run three additional regression models in which we distinguish between low-, medium-, and high-ability players (Models 3-5). To investigate whether effort contingencies also depend on the other player's type, instead of using the lagged average efforts of all own group members, in models 3-5 we include player specific lagged efforts (Low-ability effort $t-1$, Medium-ability effort $t-1$, High-ability effort $t-1)$. In all regressions we include a Period variable to capture learning/general time trends and a dummy variable indicating whether the own group won or lost the contest in the previous period (Win $t-1)$.

The regression results are reported in Table 3 . We start by discussing the results from our first two models investigating aggregate behavior in homogeneous and heterogeneous groups. In both cases, players condition their effort provision on that of their own group members (Ingroup effort $t-1$ ) as well as on the behavior of their opponent (Outgroup effort $t-1$ ). Both of these effects are highly significant. While in symmetric contests the magnitude of these effects is very similar across homogeneous and heterogeneous groups, it seems that when the contest becomes asymmetric, the two group types adjust behavior differently. In homogeneous groups, facing a heterogeneous group as an opponent leads to strengthened collaboration within groups (as indicated by the positive significant Ingroup effort $t-1 \times$ Asymmetric interaction term). Contrary to that, in heterogeneous groups we observe a diminished degree of interdependency of efforts as indicated by the negative coefficients of the Ingroup effort $t-1 \times$ Asymmetric and Outgroup effort $t-1 \times$ Asymmetric interaction terms, although only the latter effect is significant. 
Table 3 Determinants of individual efforts by group and player type

Dependent variable: Effort in period $t$ Homogene- Heterogeneous groups ous groups

All Low

Medium

High

(1)

(2)

(3)

(4)

(5)

Ingroup effort $t-1$

$\begin{array}{ll}0.137 * * * & 0.157 \text { *** } \\ (0.023) & (0.023) \\ 0.057 * & -0.037 \\ (0.034) & (0.035)\end{array}$

Low-ability effort $t-1$

Low-ability effort $t-1 \times$ asymmetric

Medium-ability effort $t-1$

\begin{tabular}{|c|c|c|c|c|}
\hline & & & $\begin{array}{l}0.103 * * * \\
(0.028)\end{array}$ & $\begin{array}{l}0.039 \\
(0.028)\end{array}$ \\
\hline & & & 0.008 & -0.014 \\
\hline & & & $(0.044)$ & $(0.044)$ \\
\hline & & $0.114 * * *$ & & $0.128 * * *$ \\
\hline & & $(0.032)$ & & $(0.031)$ \\
\hline & & -0.026 & & $-0.142 * * *$ \\
\hline & & $(0.048)$ & & $(0.047)$ \\
\hline & & 0.036 & $0.071 * *$ & \\
\hline & & $(0.032)$ & $(0.031)$ & \\
\hline & & 0.017 & 0.038 & \\
\hline & & $(0.046)$ & $(0.044)$ & \\
\hline $0.062 * * *$ & $0.075^{* * *}$ & $0.047 * * *$ & $0.088 * * *$ & $0.084 * * *$ \\
\hline (0.009) & $(0.009)$ & $(0.016)$ & $(0.015)$ & $(0.015)$ \\
\hline-0.006 & $-0.026^{* *}$ & -0.022 & $-0.049 * *$ & -0.003 \\
\hline$(0.014)$ & $(0.013)$ & $(0.022)$ & $(0.021)$ & $(0.021)$ \\
\hline$-1.413^{* * *}$ & $-1.702 * * *$ & $-2.775^{* * *}$ & $-1.729 * *$ & -0.504 \\
\hline (0.535) & $(0.512)$ & $(0.907)$ & $(0.870)$ & $(0.874)$ \\
\hline$-0.319 * * *$ & $-0.305^{* * *}$ & $-0.424 * * *$ & $-0.205^{* * *}$ & $-0.288 * * *$ \\
\hline (0.023) & $(0.023)$ & $(0.039)$ & $(0.039)$ & $(0.039)$ \\
\hline $6.186^{*}$ & 4.781 & 1.836 & 3.096 & $9.136^{*}$ \\
\hline (3.453) & (3.101) & $(4.566)$ & (5.187) & (5.493) \\
\hline $23.054 * * *$ & $24.245^{* * *} *$ & $29.295 * * *$ & $21.065 * * *$ & $22.177 * * *$ \\
\hline$(2.270)$ & (2.110) & (3.197) & $(3.593)$ & (3.904) \\
\hline Yes & Yes & Yes & Yes & Yes \\
\hline Yes & Yes & No & No & No \\
\hline Yes & Yes & Yes & Yes & Yes \\
\hline 808 & 5544 & 1848 & 1848 & 1848 \\
\hline
\end{tabular}

Medium-ability effort $t-1 \times$ asymmetric

High-ability effort $t-1$

High-ability effort $t-1 \times$ asymmetric

Outgroup effort $t-1$

Outgroup effort $t-1 \times$ asymmetric

Win $t-1$

Period

Asymmetric

1 if contest is asymmetric, 0 otherwise

Constant

Random intercepts:

Contest

Group

Subject

Observations

5808

Multilevel linear mixed-effects models using random intercepts for matching groups (a contest between two groups), groups, and individuals. In models (3)-(5), the random intercepts for groups are removed as we only consider one specific player within a group. Numbers in parentheses indicate standard errors

Significance levels $* p<0.1, * * p<0.05, * * * p<0.01$ 
To better understand these differences between homogeneous and heterogeneous groups, models 3-5 investigate type-specific behavior in heterogeneous groups. The results reveal several interesting patterns. In particular, they show that while low-ability group members condition their effort on the medium ability member's past behavior (with the magnitude being comparable to the one observed in homogeneous groups), they largely ignore the efforts of their high-ability group member. Similarly, the high-ability player largely ignores the efforts by the low-ability player, but responds to the effort of the medium-ability player, at least when the contest is symmetric (as indicated by the positive and significant Medium-ability effort $t-1$ coefficient). When the contest is asymmetric, however, high-ability players stop conditioning their efforts on those of the medium-ability player (the joint effect of the Medium-ability effort $t-1$ and the Medium-ability effort $t-1 \times$ Asymmetric coefficient is close to and not significantly different from zero, $p=0.686$ ), but instead increase their efforts unconditionally as indicated by the positive and significant Asymmetric dummy. The medium-ability player, in contrast, reacts to the lagged efforts of both the low-ability and the high-ability player irrespective of the type of contest, revealing that she plays a key role in determining the levels of within-group cooperation.

Taken together, these results suggest that the different players react very differently to the effort of others, depending on the other player's type, and that these effects seem to be contest-specific. This highlights that, as in Reuben and Riedl (2013), heterogeneity within groups can lead to a multiplicity of (potentially conflicting) behavioral norms and rules that, as we show here, might additionally depend on situational factors such as the opponent's type in a contest. We summarize these findings in our fourth result:

\section{Result 4}

1. In homogeneous groups asymmetric contests leads to an increase of conditional cooperation among group members, while no such effect is observed for heterogeneous groups.

2. In the latter, medium ability players play the key role in determining effort levels within groups as they react to both other player's behavior and vice versa. In contrast, low and high ability players largely ignore each other's efforts.

\subsection{Efficiency and inequality}

In this section, we investigate the consequences of the observed behavior on overall welfare. Given the structure of the Tullock contest, higher efforts are inevitably associated with lower earnings for the contest participants. From their point of view, the social optimum is reached when neither group invests anything into the contest (in this case the winner is determined by a coin flip).

A second important dimension, along with individual efficiency, is how the gains from competition are distributed. Results from previous research show that many 
people care about relative earnings (Sobel 2005; Fehr and Schmidt 2006). In our setting, group members in all treatments always receive an equal share of the winning prize independent of their own effort. Because marginal costs of effort are also identical for all players, equality in individual earnings can only be reached when all group members exert the same level of effort. Yet, given the different abilities (and hence different marginal productivities) of players in heterogeneous groups, standard theory predicts some amount of inequality in equilibrium as only high-ability players are predicted to exert any effort. For homogeneous groups, on the other hand, theory remains silent on the degree of inequality, as it only makes predictions on the aggregate level of contributions, but not on the division of labor within groups.

Figure 3 shows, for each group and contest type, average individual earnings (left panel) and the average degree of inequality, as measured by the standard deviation of earnings within a group in a given period (right panel). Surprisingly, despite the different group structure and distribution of ability types, when comparing homogeneous (light bars) and heterogeneous groups (grey bars) we find very similar levels of earnings and inequality at the aggregate level (earnings: 124.3 vs. $122.5, p=0.901$; inequality: 17.7 vs. 17.2, $p=0.866$ ), as well as when comparing both group types separately for symmetric and asymmetric contests (all $p>0.281$, linear mixed effects models, see Table A4 in Electronic Supplementary Material in Appendix A).

The type of contest, in contrast, has a strong impact on efficiency and the distribution of wealth. Compared to symmetric contests, asymmetric contests not only have detrimental effects on individual earnings (due to the increased efforts as highlighted in Sect. 4.1), but also on inequality. While earnings significantly decrease on average by about 5\% from 125.6 to 119.0 tokens (linear mixed effects model, $p=0.058$, see Table A6 in Electronic Supplementary Material in Appendix A), inequality significantly increases by $23 \%$ from 16.2 to $20.0(p=0.036)$. As shown by Fig. 3, while these effects occur in both types of groups, the effect of decreased earnings is particularly pronounced for homogeneous groups $(-8 \%, p=0.026$, compared to $-2 \%, p=0.524$, in heterogeneous groups), and the effect of increased inequality is particularly pronounced in heterogeneous groups $(+30 \%, p=0.049$, compared to $+17 \%, p=0.226$, in homogeneous groups; see also Table A5 in Electronic Supplementary Material in Appendix A).

Yet, in heterogeneous groups the level of inequality is still much lower than what would be observed if only the high-ability player contributes (as predicted by standard theory). In the following, we calculate how much resources heterogeneous groups forgo by adopting an equal rather than optimal sharing rule. The amount is substantial. In symmetric contests, heterogeneous groups invest on average 79.8 tokens, leading to contributions (effort $\times$ ability) of 160.3 tokens. The same amount of contributions, however, could have also been achieved if the high-ability player invested only 53.4 tokens. Heterogeneous groups thus could have spent about one third less of what they actually did without changing the overall group contributions. Of course, this would have led to a considerable increase in within-group inequality (at the expense of high-ability players). Similar amounts are observed in asymmetric contests, where heterogeneous groups use $45 \%$ more resources than would have been optimal. We summarize these findings in our fifth result: 

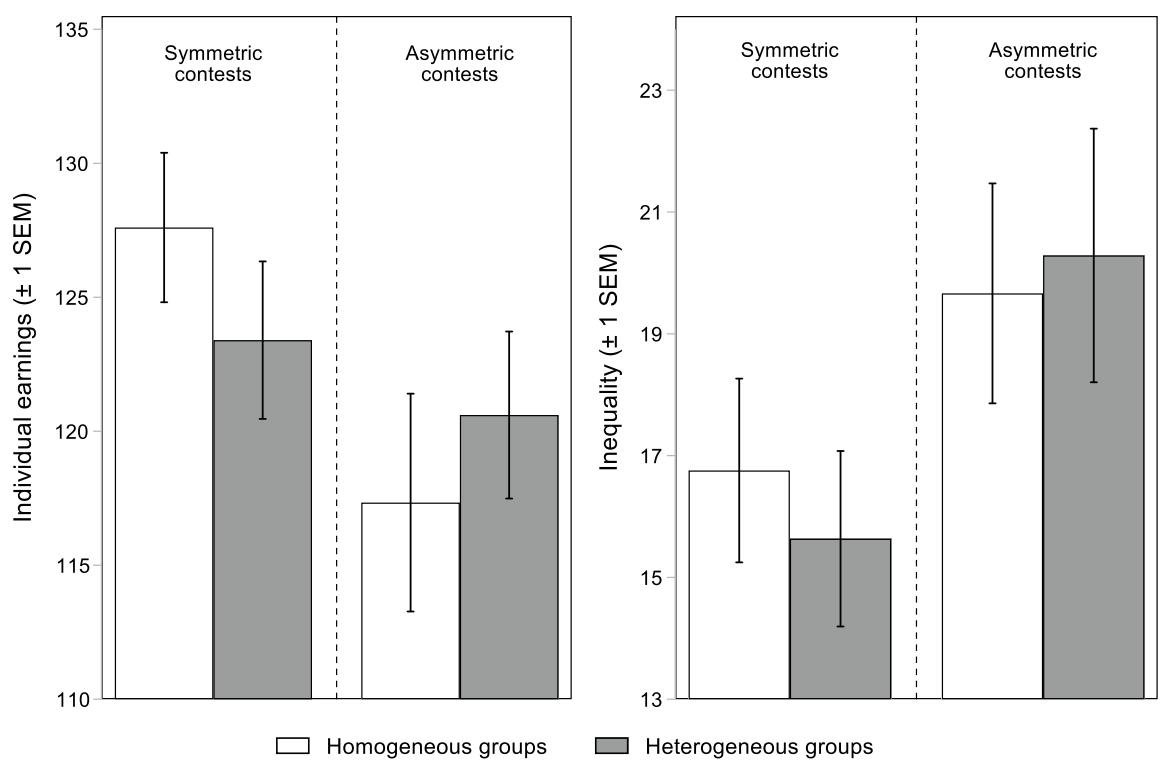

Fig. 3 Earnings and inequality by group and contest type

Result 5 Compared to symmetric contests, asymmetric contests decrease individual earnings and increase inequality within groups. In heterogeneous groups inequality levels are much lower than predicted by theory and groups forgo a substantial amount of wealth by sharing labor equally rather than optimally.

\section{Equity norm model}

The results of our experiment constitute a challenge for the theoretical predictions reviewed in Sect. 2 (see Table 1). While the joint payoff maximization model does better than the standard and the parochial altruism models in predicting the aggregate intensity of competition, all models fail to predict the comparative statics that we observe, particularly because they all predict very different behavior for top, medium, and low ability players in heterogeneous groups, and do not anticipate that competition will be more intense in asymmetric contests, particularly for homogeneous groups.

In this section, we discuss the predictions of an equity norm model that can reconcile our results with a theoretical rationale. The model is based on a variant of the inequity aversion model developed by Fehr and Schmidt (1999), and on a more recent social norm compliance model by Fehr and Schurtenberger (2018). These models have been used to explain empirical regularities in a variety of games such as cooperative behavior in social dilemma games, giving behavior in dictator games, and reward and punishment behavior in trust and ultimatum games (see e.g., Sobel 2005; Cooper and Kagel 2016, for overviews), but, so far, have not been applied 
to group contests. Yet, our results, as well as evidence from previous experimental studies, indicate that other-regarding concerns may affect behavior in this setting as well. Specifically, as highlighted here as well as by, e.g., Abbink et al. (2010), in group contests individuals condition their effort choices on those of other group members, even when efforts are perfect substitutes and overall effort levels are above the standard equilibrium prediction. Such coordination behavior is consistent with the notion that individuals have a preference for (partially) matching their peers' efforts. $^{15}$

To capture this, the main assumption of our equity norm model is that individuals derive a disutility from investing less than the average of the other group members (which we interpret as a signal of normative behavior). Assuming risk neutrality, the expected utility of player $i$ in group $j$ can then be written as follows:

$$
U_{i, j}\left(x_{i, j}, X_{1}, X_{2}\right)=\pi_{i}-\tau_{i} \cdot \max \left\{\bar{E}_{-i, j}-e_{i, j}, 0\right\}
$$

The first part of player $i$ 's utility function is simply given by her expected monetary payoff, $\pi_{i}$ (see Sect. 2). The second term is the nonpecuniary part of the utility function, which depends on player $i$ 's concern for equity, $\tau_{i}$, and the difference between the others' average effort and player $i$ 's effort $\left(\bar{E}_{-i, j}-e_{i, j}\right)$. The equity parameter $\tau_{i} \geq 0$ captures player $i$ 's marginal disutility from exerting one unit of effort less than the other group members. This disutility can be interpreted as, e.g., guilt from contributing less than others (as in Fehr and Schmidt 1999), or the psychological cost of deviating from the norm (as in Fehr and Schurtenberger 2018). Intuitively, the larger $\tau_{i}$, the larger is $i$ 's disutility from contributing less than her peers, and, hence, the larger her incentive to match the effort level of her group members. When $\tau_{i}=0$ there is no disutility from contributing less than the others; in this case players care only about their own monetary payoff.

While the empirical validity of this model is appealing, it is not straightforward to make behavioral predictions based on it. Even in much simpler settings than ours, the model above (or variants thereof) typically leads to a multiplicity of equilibria (see e.g. Fehr and Schmidt 1999). The context of group contests, as considered here, is no exception; The equity component increases the complexity of the bestresponse functions, generating a multiplicity of equilibria in both symmetric and asymmetric contests, making it hard to provide a straightforward analytical solution. We can, however, calculate numerical solutions. Table 4 shows the outcome of such a numerical exercise, making separate predictions for symmetric and asymmetric contests, as well as for homogeneous and heterogeneous groups. For our calculations, we use two different values for the equity parameter, one low $(\tau=0.2)$ and one $\operatorname{high}(\tau=0.7) .{ }^{16}$

\footnotetext{
15 Such coordination attempts were particularly easy in our context, as at the end of each period, players received detailed feedback about the effort, the contributions, and the earnings of all group members.

16 Table 4 shows the range of equilibrium efforts. Figure A3 and Table A6 in the Electronic Supplementary Material in appendix characterize the best response functions and the equilibrium outcomes for each group and condition. Note that while in practice $\tau$ is an individual-level parameter that might differ across participants, for simplicity, in the following, we assume $\tau$ to be identical for all players and that this is common knowledge.
} 


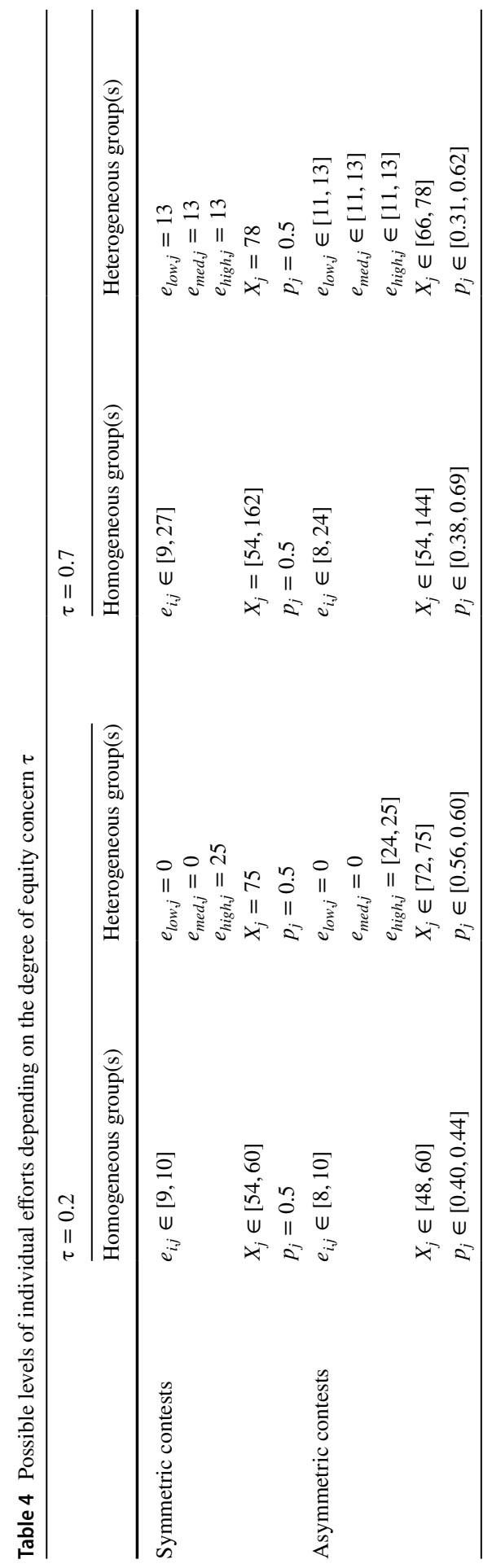


We start by discussing the effects that equity concerns have on the distribution of labor within groups. As shown in Table 4, when all group members have the same ability (homogeneous groups), even a relatively low degree of equity concerns is sufficient to guarantee uniform efforts by all group members (recall that in the standard case $(\tau=0)$ any combination of efforts that leads to the unique aggregate equilibrium effort is an equilibrium). The reason is that a negative deviation from others' average effort now creates a psychological cost due to equity concerns. When all group members have the same marginal material incentive to exert effort, this additional psychological cost is sufficient to solve the free rider problem within groups, as players prefer to match the effort of their peers in order to avoid the inequity costs.

When group members differ in their ability (heterogeneous groups), in contrast, the prediction for a low degree of the equity concerns $(\tau=0.2)$ is the same as in the standard case: only the group member with the highest ability exerts any effort, while the others free ride. If the concern for equity is sufficiently strong $(\tau=0.7)$, however, the model predicts uniform efforts even in heterogeneous groups, despite the players' different abilities. The reason is that the different material incentives to provide effort (based on the players' marginal productivity) are counterbalanced by the disutility that is generated when group members exert different levels of effort. But the latter effect has to be strong enough for players to match each other's effort despite their different abilities.

We now turn to the question of how equity concerns affect the overall level of effort at the aggregate level. As can be seen in Table 4, the equity norm model predicts a vast range of equilibria, especially for high levels of $\tau$ and in particular with regard to the effort of homogeneous groups. For example, in symmetric contests between two homogeneous groups, for $\tau=0.7$ any (symmetric) effort profile from 9 to 27 is an equilibrium in pure strategies of the stage game. The intuition here is simple but challenging. For any of these symmetric effort profiles, the increased probability of winning associated with a unilateral increase in effort is not worth its cost, and a unilateral decrease in effort is penalized by both a lower probability of victory and the inequity aversion disutility. The multiple equilibria predicted for homogeneous groups simply acknowledge that this logic applies for very different effort levels, from a lower bound close to the standard Nash prediction (9), up to three times that amount (27). A similar large range is predicted for homogeneous groups in asymmetric contests, where individual equilibrium efforts range from 8 to 24.

For heterogeneous groups, the model makes a unique aggregate effort prediction when the contest is symmetric, but not when it is asymmetric. In the latter case, multiple equilibria exist as well, as the range of possible contest expenditures by the homogeneous groups is vast. In Fig. A3 in Electronic Supplementary Material in Appendix A we plot the reaction functions of homogeneous and heterogeneous groups for $\tau=0.2$ (panel (a)) and $\tau=0.7$ (panel (b)).

Given the multiplicity of equilibria, without further assumptions it is a priori unclear on which equilibrium participants will coordinate on, leaving us with a 
complex equilibrium selection problem. To identify which equilibria may be more prominent than other, in the following we consider the well-established refinement criteria of Pareto dominance. That is, while (by definition) in all equilibria no individual has an incentive to deviate unilaterally, we assume that a group would prefer to jointly deviate to the equilibrium that yields the highest payoff.

Applying this refinement criterion to our predictions above, we find that for symmetric contests the Pareto criterion drives players towards lower effort levels. The reason is that, because in symmetric contests both groups always exert the same effort, in equilibrium the winning probability is always equal to $50 \%$. As a result, higher efforts only lead to higher costs but not to higher expected benefits, and, therefore, payoffs are highest when efforts are lowest. In asymmetric contests, in contrast, the same logic no longer applies, as efforts by homogeneous and heterogeneous groups may differ. Here, the maximum joint payoff for homogeneous groups is reached for effort levels of either 22 (when each member of the heterogeneous group contributes 13 or 11) or 23 (when the individual efforts of the opponent is 12). Therefore, this scenario allows for the homogeneous group to be more aggressive than their heterogeneous opponent, and have a higher probability of winning than what is predicted by the standard model. ${ }^{17}$

\section{Conclusion}

Some people are stronger, smarter, or wealthier than others. An obvious consequence is that the ability to perform particular tasks is not identical for everyone. In this light, research on contests that presupposes identical abilities is rather idiosyncratic. To address this issue, the current work investigates the effects of withingroup heterogeneity in abilities on behavior in inter-group contests. We compare the commonly explored symmetric contests between homogeneous groups-in which the ability is identical for all group members in both groups-to symmetric contests between two heterogeneous groups, and to asymmetric contests between homogeneous and heterogeneous groups.

Our main result is that while heterogeneity within groups per se has no discernable effect on competition, asymmetry between groups leads to a significant intensification and increased volatility of conflict. This effect is driven by homogeneous and heterogeneous groups who both increase their efforts relative to the symmetric contest case. As a result, heterogeneous groups do not utilize their predicted advantage when competing against homogeneous groups, as both groups win the contest equally often.

One reason for this result is that from an efficiency point of view, heterogeneous groups share the labor in a suboptimal manner. In particular, in contrast to standard theory which predicts that only the group member with the highest ability should exert effort, while all others free ride, we find that efforts of low-, medium-, and

17 See also Table A6 in Electronic Supplementary Material in Appendix A which presents the outcome of equilibrium selection using the Pareto criterion when $\tau=0.7$. 
high-ability players are almost identical, especially in symmetric contests. This indicates that, in line with a notion of fairness, high-ability players are not willing to accept the relatively lower payoffs that are inevitably associated with exerting more effort than the other group members, nor do the less able group members expect them to do so. ${ }^{18}$ Such equal sharing of labor is somewhat attenuated when heterogeneous groups compete against homogeneous groups in asymmetric contests. In this case, high-ability players invest more effort despite their less able peers not willing to match these increased contributions. It thus seems that in asymmetric contests highability players understand the comparative advantage they have and are more willing to accept the responsibility that comes with being the most able member of the group. Yet, the distribution of efforts is still far from the standard theoretical predictions, and homogeneous and heterogeneous groups share the burden of effort provision in a remarkably similar fashion, despite their very different structure. Interestingly, while in heterogeneous groups we observe a weaker interdependency of efforts between group members when the contest becomes asymmetric, the opposite pattern is observed in homogeneous groups where conditional cooperation is increased when the opponent group is different from the own. The latter effect might be due to an increased sense of identity that is triggered by facing a different group (Chowdhury et al. 2016), or due to an increased sense of threat triggered by the presence of a highability member in the opposing group. Alternatively, as shown by Hargreaves Heap et al. (2015) in a related context, in asymmetric contests disadvantaged groups might react doggedly by increasing their efforts when facing a stronger opponent.

In Sect. 5, we proposed an equity norms model that can reconcile our findings above, at least qualitatively. More research is needed, however, to further understand individual motivations and group dynamics in these contexts. Furthermore, while our laboratory experiment provided only a minimal environment for analyzing the effects of heterogeneity in symmetric and asymmetric contest, we believe that more research is needed in order to test the robustness of our results in more complex and rich environments, e.g., in natural field settings. Another interesting avenue for future research is to investigate whether common institutions such as communication, leadership, or punishment, which have been found to be effective in increasing cooperation in homogeneous groups (e.g., Abbink et al. 2010; Leibbrandt and Sääksvuori 2012; Cason et al. 2012; Eisenkopf 2014), are similarly effective in heterogeneous groups.

Open Access This article is licensed under a Creative Commons Attribution 4.0 International License, which permits use, sharing, adaptation, distribution and reproduction in any medium or format, as long as you give appropriate credit to the original author(s) and the source, provide a link to the Creative Commons licence, and indicate if changes were made. The images or other third party material in this article are included in the article's Creative Commons licence, unless indicated otherwise in a credit line to the material. If material is not included in the article's Creative Commons licence and your intended use is not permitted by statutory regulation or exceeds the permitted use, you will need to obtain permission directly from the copyright holder. To view a copy of this licence, visit http://creativecommons.org/licenses/by/4.0/.

18 These results are in line with evidence from public goods experiments, which also find a high degree of interdependency between contributions of group members of different abilities (Kölle 2015). 


\section{References}

Abbink, K., Brandts, J., Herrmann, B., \& Orzen, H. (2010). Intergroup conflict and intra-group punishment in an experimental contest game. American Economic Review, 100, 420-447.

Ahn, T. K., Isaac, R. M., \& Salmon, T. C. (2011). Rent seeking in groups. International Journal of Industrial Organization, 29(1), 116-125.

Amaldoss, W., \& Staelin, R. (2010). Cross-function and same-function alliances: how does alliance structure affect the behavior of partnering firms? Management Science, 56(2), 302-317.

Baik, K. H. (2008). Contests with group-specific public-good prizes. Social Choice and Welfare, 30(1), $103-117$.

Bandiera, O., Barankay, I., \& Rasul, I. (2013). Team incentives: Evidence from a firm level experiment. Journal of the European Economic Association, 11(5), 1079-1114.

Bernhard, H., Fischbacher, U., \& Fehr, E. (2006). Parochial altruism in humans. Nature, 442(7105), 912.

Bhattacharya, P. (2016). Inter-team contests with power differential. Journal of Economic Behavior \& Organization, 132, 157-175.

Bolton, G. E., \& Ockenfels, A. (2000). ERC: A theory of equity, reciprocity, and competition. American Economic Review, 90(1), 166-193.

Brandts, J., Cooper, D. J., \& Fatas, E. (2007). Leadership and overcoming coordination failure with asymmetric costs. Experimental Economics, 10(3), 269-284.

Brandts, J., Cooper, D. J., Fatas, E., \& Qi, S. (2016). Stand by me: Help, heterogeneity and commitment in experimental coordination games. Management Science, 62, 2916-2936.

Brookins, P., Lightle, J. P., \& Ryvkin, D. (2015). An experimental study of sorting in group contests. Labour Economics, 35, 16-25.

Brookins, P., \& Ryvkin, D. (2014). An experimental study of bidding in contests of incomplete information. Experimental Economics, 17(2), 245-261.

Cason, T. N., Sheremeta, R. M., \& Zhang, J. (2012). Communication and efficiency in competitive coordination games. Games and Economic Behavior, 76(1), 26-43.

Cason, T. N., Sheremeta, R. M., \& Zhang, J. (2017). Asymmetric and endogenous within-group communication in competitive coordination games. Experimental Economics, 20, 946-972.

Chen, H., Ham, S. H., \& Lim, N. (2011). Designing multiperson tournaments with asymmetric contestants: An experimental study. Management Science, 57(5), 864-883.

Choi, J. K., \& Bowles, S. (2007). The coevolution of parochial altruism and war. Science, 318(5850), $636-640$.

Chowdhury, S. M., Lee, D., \& Topolyan, I. (2016). The max-min group contest: weakest-link (group) allpay auction. Southern Economic Journal, 83, 105-125.

Cooper, D. J., \& Kagel, J. H. (2016). Other-regarding preferences. The Handbook of Experimental Economics, 2, 217.

Croson, R., Fatas, E., \& Neugebauer, T. (2005). Reciprocity, matching and conditional cooperation in two public goods games. Economics Letters, 87(1), 95-101.

Croson, R., Fatas, E., Neugebauer, T., \& Morales, A. J. (2015). Excludability: A laboratory study on forced ranking in team production. Journal of Economic Behavior \& Organization, 114, 13-26.

Dechenaux, E., Kovenock, D., \& Sheremeta, R. M. (2015). A survey of experimental research on contests, all-pay auctions and tournaments. Experimental Economics, 18(4), 609-669.

Eisenkopf, G. (2014). The impact of management incentives in intergroup contests. European Economic Review, 67, 42-61.

Fallucchi, F., Renner, E., \& Sefton, M. (2013). Information feedback and contest structure in rent-seeking games. European Economic Review, 64, 223-240.

Fehr, E., \& Schmidt, K. M. (1999). A theory of fairness, competition, and cooperation. Quarterly Journal of Economics, 114(3), 817-868.

Fehr, E., \& Schmidt, K. M. (2006). The economics of fairness, reciprocity and altruism-experimental evidence and new theories. Handbook of the Economics of Giving, Altruism and Reciprocity, 1, 615-691.

Fehr, E., \& Schurtenberger, I. (2018). Normative foundations of human cooperation. Nature Human Behaviour, 2(7), 458-468.

Fischbacher, U. (2007). z-Tree: Zurich toolbox for ready-made economic experiments. Experimental Economics, 10(2), 171-178. 
Fonseca, M. A. (2009). An experimental investigation of asymmetric contests. International Journal of Industrial Organization, 27(5), 582-591.

Hamilton, B. H., Nickerson, J. A., \& Owan, H. (2003). Team incentives and worker heterogeneity: An empirical analysis of the impact of teams on productivity and participation. Journal of Political Economy, 111(3), 465-497.

Hargreaves Heap, S. P., Ramalingam, A., Ramalingam, S., \& Stoddard, B. V. (2015). "Doggedness" or "disengagement"? An experiment on the effect of inequality in endowment on behaviour in team competitions. Journal of Economic Behavior \& Organization, 120, 80-93.

Katz, E., Nitzan, S., \& Rosenberg, J. (1990). Rent-seeking for pure public goods. Public Choice, 65(1), 49-60.

Kölle, F. (2015). Heterogeneity and cooperation: The role of capability and valuation on public goods provision. Journal of Economic Behavior \& Organization, 109, 120-134.

Kölle, F., Sliwka, D., \& Zhou, N. (2016). Heterogeneity, inequity aversion, and group performance. Social Choice and Welfare, 46(2), 263-286.

Konrad, K. A. (2009). Strategy and dynamics in contests. Oxford: Oxford University Press.

Kurschilgen, M., Morell, A., \& Weisel, O. (2017). Internal conflict, market uniformity, and transparency in price competition between teams. Journal of Economic Behavior \& Organization, 144C, 121-132.

Leibbrandt, A., \& Sääksvuori, L. (2012). Communication in intergroup conflicts. European Economic Review, 56(6), 1136-1147.

Lim, W., Matros, A., \& Turocy, T. L. (2014). Bounded rationality and group size in Tullock contests: Experimental evidence. Journal of Economic Behavior \& Organization, 99, 155-167.

Nalbantian, H. R., \& Schotter, A. (1997). Productivity under group incentives: An experimental study. The American Economic Review, 87(3), 314-341.

Orrison, A., Schotter, A., \& Weigelt, K. (2004). Multiperson tournaments: An experimental examination. Management Science, 50(2), 268-279.

Rapoport, A., Bornstein, G., \& Erev, I. (1989). Intergroup competition for public goods: Effects of unequal resources and relative group size. Journal of Personality and Social Psychology, 56(5), 748.

Reuben, E., \& Riedl, A. (2013). Enforcement of contribution norms in public good games with heterogeneous populations. Games and Economic Behavior, 77(1), 122-137.

Ryvkin, D. (2011). The optimal sorting of players in contests between groups. Games and Economic Behavior, 73(2), 564-572.

Sääksvuori, L., Mappes, T., \& Puurtinen, M. (2011). Costly punishment prevails in intergroup conflict. Proceedings of the Royal Society of London B: Biological Sciences, 278(1723), 3428-3436.

Sheremeta, R. M. (2010). Experimental comparison of multi-stage and one-stage contests. Games and Economic Behavior, 68(2), 731-747.

Sheremeta, R. M. (2011a). Contest design: An experimental investigation. Economic Inquiry, 49(2), 573-590.

Sheremeta, R. M. (2011b). Perfect-substitutes, best-shot, and weakest-link contests between groups. Korean Economic Review, 27, 5-32.

Sheremeta, R. M. (2017). Behavior in group contests: A review of experimental research. Journal of Economic Surveys, 32, 683-704.

Sobel, J. (2005). Interdependent preferences and reciprocity. Journal of Economic Literature, 43(2), $392-436$.

Tullock, G. (1980). Efficient rent seeking. In J. Buchanan, R. Tollison, \& G. Tullock (Eds.), Towards a theory of the rent-seeking society (pp. 97-112). College Station: Texas A\&M Press.

Publisher's Note Springer Nature remains neutral with regard to jurisdictional claims in published maps and institutional affiliations. 


\section{Affiliations}

\section{Francesco Fallucchi ${ }^{1} \cdot$ Enrique Fatas $^{2,3,4}\left(\mathbb{D} \cdot\right.$ Felix Kölle $^{5} \cdot$ Ori Weisel $^{6}$}

Francesco Fallucchi

Francesco.Fallucchi@liser.lu

Felix Kölle

felix.koelle@uni-koeln.de

Ori Weisel

oriw@tauex.tau.ac.il

1 Luxembourg Institute of Socio-Economic Research, LISER, Esch-sur-Alzette, Luxembourg

2 School of Business and Economics, Loughborough University, Loughborough, UK

3 Escuela de Administración, Universidad ICESI, Cali, Colombia

4 Center for Social Norms and Behavioral Dynamics, University of Pennsylvania, Philadelphia, USA

5 Department of Economics, University of Cologne, Cologne, Germany

6 Organizational Behavior Group, Coller School of Management, Tel Aviv University, Tel Aviv, Israel 